\title{
On Optimal Favoritism in Asymmetric Competitions
}

\author{
David Lu \\ United World College of South East Asia, Singapore \\ Email:1u12753@gapps.uwcsea.edu.sg
}

How to cite this paper: Lu, D. (2021). On Optimal Favoritism in Asymmetric Competitions. Theoretical Economics Letters, 11, 74-83.

https://doi.org/10.4236/tel.2021.111006

Received: December 2, 2020

Accepted: February 7, 2021

Published: February 10, 2021

Copyright $\odot 2021$ by author(s) and Scientific Research Publishing Inc. This work is licensed under the Creative Commons Attribution International License (CC BY 4.0).

http://creativecommons.org/licenses/by/4.0/

\begin{abstract}
Favoritism towards a relatively weaker competitor is widely adopted as an effective instrument to enhance productive effort provision in asymmetric competitions. In this paper, we investigate the effort-maximizing favoritism rule in asymmetric two-player contests with all-pay auction technology, while accommodating fully flexible (nonlinear) favoritism rules. We assume that the players' competencies (measured by their values of winning the competition, or marginal effort costs) are public information. We find that at the optimum, the weaker player is extremely favored; however his/her winning chance converges to zero. This finding illustrates that the effort-maximizing extreme favoritism rule perversely decreases winner diversity.
\end{abstract}

\section{Keywords}

Affirmative Action, All-Pay Auction, Asymmetric Contest, Favoritism

\section{Introduction}

Economic, political, social and athletic competitions, in which the contestants are asymmetric, are abundant. In $\mathrm{R} \& \mathrm{D}$ races, innovators are typically endowed with different technological capacities and expertise. In government procurements, suppliers (say domestic versus foreign firms) can be heterogeneous in their provision efficiencies. In job promotions within hierarchical organizations, contenders usually differ in their competitiveness. In political campaigns, some candidates are more popular or less financially constrained compared to their opponents. In school admissions, applicants come from diverse racial, economic and social and backgrounds. In athletic events, such as golf and horse racing, players essentially differ in their levels of training, field experiences and physical conditions. 
It has long been recognized that heterogeneity in contestants' competitiveness can hinder their incentive to exert high effort overall. For example, one notable empirical study by Brown (2011) convincingly shows that average score of golf players falls in the presence of a superstar like Tiger Woods. The intuition behind this finding is clear: with the presence of a dominant opponent, weaker players have less incentive to exert effort due to the slim chance of winning the grand award. Given this, the superstar does not need to exert much effort to win.

This undesirable discouragement effect of player heterogeneity on total effort supply has stimulated enormous academic interest on investigating how to mitigate or overcome it. A well received insight from literature is that leveling the battle field by favoring the weaker player is essential to encourage the weaker and discipline the stronger. In other words, introducing appropriate favoritism, say head start and/or handicap, in the originally asymmetric competition can mitigate the discouragement effect of player heterogeneity. Dragov and Ryvkin (2017) further find that in some settings biased contests of symmetric players can also be optimal in terms of various objectives including expected aggregate effort. On the other hand, the existence of discouragement effect is examined more carefully by Dragov and Ryvkin (2020), who find that the effect might not always exist.

In this paper, we further investigate the effort-maximizing favoritism rule while adopting an analytical framework of two-player all pay auction with complete information. Differentiating from previous literature, we allow fully nonlinear favoritism rules without restricting to linear instruments such as headstarts, handicaps, or a combination of the two. Specifically, we define a favoritism rule as a player's winning effort threshold as a function of the other player's effort. A player wins if and only if his/her effort is above this threshold. We will identify the optimal favoritism rule and the induced bidding equilibrium, and study the properties and implications of the optimal rule.

We find that the effort-maximizing favoritism rule would favor the weak player to the extreme. However, at the optimum, the weak player would (almost) always lose the competition. This finding reveals that the effort-maximizing extreme favoritism certainly decreases winner diversity in our setting. One implication of our finding is that the well received insight of "fully leveling the playing field" (e.g. Nti, 2004 and Fu, 2006, etc.) is no longer applicable once nonlinear favoritism rules are allowed.

The insight of using favoritism to better incentivize the contestants is corroborated by an established line of theoretical studies, which provide sound economic justification for policies and practices that specify preferential treatment of contestants in asymmetric competitions. The most salient example is affirmative action in school admissions (see Fu, 2006; Franke, 2012). Epstein et al. (2011) emphasize on the benefit of public policies that favor small and medium-sized firms in government procurements. Preferential policies are often observed in sports. For example, to make the competition more exciting, higher 
ranked competitors are often handicapped in golf and horse racing (Chowdhury et al., 2019). Che and Gale (1998) establish that with asymmetric contestants, it is optimal to handicap the most efficient to boost their overall performance. This insight echoes the rationale for the research recognition programs, where young researchers obtain head-starts (Kirkegaard, 2012).

Besides studies mentioned above, this line of the literature on favoritism also includes Nti (2004), Sahuguet (2006), Tsoulouhas et al. (2007), Franke et al. (2013), Seel \& Wasser (2014), Franke et al. (2018), and Zhu (2019) among many others. Unlike our paper which allows nonlinear favoritism rules, the favoritism in these existing studies focuses on linear instruments, including head start (an additive bias on a player's performance/effort) and handicap (a multiplicative bias on a player's performance/effort). Our study further strengthens these studies by providing the fully effort-maximizing favoritism rule without imposing any restrictions.

Favoritism towards weaker competitors can also promote the diversity of winner group, as illustrated by Fu (2006), Pastine \& Pastine (2012) and Lee (2013). These studies nevertheless also find that there can be a tension between effort-maximization and winner diversity when the contest organizer is restricted to adopt linear favoritism instruments. Our findings further complement these studies by revealing that effort-maximizing favoritism that extremely favor the weaker player can indeed perversely reduce the diversity of winner group. This result clearly has important policy implications, since preferential policies including affirmative actions typically aim at boosting winner diversity.

The rest of the paper is organized as follows. In Section 2, we set up the model and carry out the analysis. Section 3 provides some concluding remarks.

\section{Model Setup and Analysis}

We adopt an analytical framework of all pay auction with complete information. There are two players $i=1,2$. The players' competencies can be measured by their values of winning the competition, or equivalently their marginal effort costs. Throughout the paper, we use values of winning to measure players' competencies. Alternatively, if we adopt a setting in which players' marginal effort costs are used to measure their competencies, we can assume that their marginal effort costs are reciprocals of their winning values. The analysis would be identical.

Without loss of generality, we assume $v_{1}>v_{2}$, i.e. bidder 1 is a stronger bidder. Bidder $i \mathrm{~s}$ bid/effort is denoted by $b_{i} \geq 0, i=1,2$. The higher bidder wins and both bidders incur the cost of their bid, which is $b_{i}$. The bidder with higher value wins when there is a tie in their bids.

Definition 1. The favoritism rule is specified by bidder 2's winning threshold $B\left(b_{1}\right) \in\left[0, v_{2}\right]$, which is a strictly increasing and continuous function defined on $\left[0, \bar{b}_{1}\right]$, where $\bar{b}_{1} \in\left(0, v_{1}\right]$. This rule means that bidder 2 with bid $b_{2}$ wins if and only if his bid $b_{2}$ is no less than $B\left(b_{1}\right)$ when bidder 1 places bid $b_{1} \in\left[0, \bar{b}_{1}\right]$. We use $B^{-1}\left(b_{2}\right)$ to denote the inverse function of $B\left(b_{1}\right)$. 
We first provide a general result of equilibrium construction under an arbitrary favoritism rule, which is defined as above.

Proposition 1. Consider favoritism rule $B\left(b_{1}\right)$ such that $B(0)=0$ and $B\left(\bar{b}_{1}\right)=v_{2}$ where $\bar{b}_{1} \in\left[v_{2}, v_{1}\right]$. Then the following is a mixed strategy equilibrium:

$$
\begin{aligned}
& F_{1}\left(b_{1}\right)=\frac{B\left(b_{1}\right)}{v_{2}}, b_{1} \in\left[0, \bar{b}_{1}\right] \\
& \text { and } F_{2}\left(b_{2}\right)=\frac{v_{1}-\bar{b}_{1}}{v_{1}}+\frac{B^{-1}\left(b_{2}\right)}{v_{1}}, b_{2} \in\left[0, v_{2}\right]
\end{aligned}
$$

At equilibrium, bidder 1 enjoys an expected payoff of $v_{1}-\bar{b}_{1}$; and bidder 2 has an expected payoff of zero.

Proof. Given bidder 2 plays $F_{2}\left(b_{2}\right)$ and the favoritism rule $B(\cdot)$, bidder 1's expected payoff is as follows if s/he bids $b_{1} \in\left[0, \bar{b}_{1}\right]$ :

$$
\pi_{1}\left(b_{1}\right)=F_{2}\left(B\left(b_{1}\right)\right) v_{1}-b_{1}=\left[\frac{v_{1}-\bar{b}_{1}}{v_{1}}+\frac{B^{-1}\left(B\left(b_{1}\right)\right)}{v_{1}}\right] v_{1}-b_{1}=v_{1}-\bar{b}_{1} .
$$

Given bidder 1 plays $F_{1}\left(b_{1}\right)$ and the favoritism rule $B(\cdot)$, bidder 2's expected payoff is as follows if s/he bids $b_{2} \in\left[0, v_{2}\right]$ :

$$
\pi_{2}\left(b_{2}\right)=F_{1}\left(B^{-1}\left(b_{2}\right)\right) v_{2}-b_{2}=\frac{B\left(B^{-1}\left(b_{2}\right)\right)}{v_{2}} v_{2}-b_{2}=0 .
$$

We next construct the following favoritism rule $B\left(b_{1}\right)$ and derive the associated bidding equilibrium $F_{i}\left(b_{i}\right), i=1,2$ by Proposition 1 .

Take small positive numbers $\varepsilon$ and $\delta$. Let

$$
\frac{B\left(b_{1}\right)}{v_{2}}= \begin{cases}\frac{b_{1}}{v_{1}-\delta} \varepsilon, & \text { if } 0 \leq b_{1} \leq v_{1}-\delta, \\ \varepsilon+\frac{1-\varepsilon}{\delta}\left[b_{1}-\left(v_{1}-\delta\right)\right], & v_{1}-\delta \leq b_{1} \leq v_{1} .\end{cases}
$$

Note $\bar{b}_{1}=v_{1}$ and $B\left(b_{1}\right) \in\left[0, v_{2}\right]$. The inverse function of $B\left(b_{1}\right)$ is denoted by

$$
\frac{B^{-1}\left(b_{2}\right)}{v_{1}}= \begin{cases}\frac{b_{2} / v_{2}}{\varepsilon}\left(1-\delta / v_{1}\right), & \text { if } 0 \leq b_{2} \leq v_{2} \varepsilon, \\ \left(1-\delta / v_{1}\right)+\frac{\delta / v_{1}}{1-\varepsilon}\left[b_{2} / v_{2}-\varepsilon\right], & \text { if } v_{2} \varepsilon \leq b_{2} \leq v_{2} .\end{cases}
$$

Define bidding strategies:

$$
F_{1}\left(b_{1}\right)=\frac{B\left(b_{1}\right)}{v_{2}} \text {, and } F_{2}\left(b_{2}\right)=\frac{B^{-1}\left(b_{2}\right)}{v_{1}} .
$$

Based on Proposition 1, we have the following result.

Corollary 1. $F_{1}\left(b_{1}\right)$ and $F_{2}\left(b_{2}\right)$ constitute a mixed strategy bidding equilibrium in the all pay auction under the favoritism rule $B(\cdot)$ in (2). 
We next establish an effort upper bound for the total expected effort induced in an all pay auction under any favoritism rule. Each bidder $i$ s expected effort equals $v_{i} \operatorname{Pr}(i$ wins $)$ minus his/her equilibrium payoff. Note either player's equilibrium payoff must be nonnegative due to their participation constraint. Therefore, the total expected effort at any bidding equilibrium must be no greater than $\sum_{i} v_{i} \operatorname{Pr}(i$ wins $)$, which is no greater than $v_{1}$ since $v_{1}>v_{2}$ and $\sum_{i} \operatorname{Pr}(i$ wins $)=1$. We thus have the following result.

Proposition 2. The total expected effort induced in an all pay auction under any favoritism rule must be no greater than $v_{1}$.

Note that at the above identified equilibrium in Corollary 1, both bidders' equilibrium payoffs are zero (by Proposition 1). Therefore, each bidder is expected effort simply equals $v_{i} \operatorname{Pr}(i$ wins $)$. We thus have the total expected effort is

$$
\begin{aligned}
T E & =v_{1}[1-\operatorname{Pr}(\text { bidder } 2 \text { wins })]+v_{2} \operatorname{Pr}(\text { bidder } 2 \text { wins }) \\
& =v_{1}+\left[v_{2}-v_{1}\right] \operatorname{Pr}(\text { bidder } 2 \text { wins }) .
\end{aligned}
$$

We next show that under favoritism rule $B(\cdot)$ in (2), the total equilibrium expected effort converges to the upper bound $v_{1}$ when $\varepsilon$ and $\delta$ converge to zero.

Proposition 3. Under favoritism rule $B(\cdot)$ in (2), the total equilibrium expected effort converges to the upper bound $v_{1}$ when $\varepsilon$ and $\delta$ converge to zero. Therefore, such favoritism rule achieves an epsilon-optimality. Moreover, the winning probability of the stronger player converges to 1.

Proof. Recall $T E=v_{1}+\left[v_{2}-v_{1}\right] \operatorname{Pr}$ (bidder 2 wins). at equilibrium. For our purpose, we only need to show that $\operatorname{Pr}$ (bidder 2 wins) converges to zero.

$$
\begin{aligned}
& \operatorname{Pr}(\text { bidder } 2 \text { wins })=\int_{0}^{v_{2}} \int_{0}^{B^{-1}\left(b_{2}\right)} \mathrm{d} F_{1}\left(b_{1}\right) \mathrm{d} F_{2}\left(b_{2}\right) \\
& =\int_{0}^{v_{2}} F_{1}\left(B^{-1}\left(b_{2}\right)\right) \mathrm{d} F_{2}\left(b_{2}\right)=\int_{0}^{v_{2}} \frac{B\left(B^{-1}\left(b_{2}\right)\right)}{v_{2}} \mathrm{~d} F_{2}\left(b_{2}\right) \\
& =\int_{0}^{v_{2}} \frac{b_{2}}{v_{2}} \mathrm{~d} F_{2}\left(b_{2}\right)=\left.\frac{b_{2}}{v_{2}} F_{2}\left(b_{2}\right)\right|_{0} ^{v_{2}}-\int_{0}^{v_{2}} \frac{1}{v_{2}} F_{2}\left(b_{2}\right) \mathrm{d} b_{2} \\
& =1-\frac{1}{v_{2}} \int_{0}^{v_{2} \varepsilon} \frac{b_{2} / v_{2}}{\varepsilon}\left(1-\delta / v_{1}\right) \mathrm{d} b_{2} \\
& \quad+\frac{1}{v_{2}} \int_{v_{2} \varepsilon}^{v_{2}}\left\{\left(1-\delta / v_{1}\right)+\frac{\delta / v_{1}}{1-\varepsilon}\left[b_{2} / v_{2}-\varepsilon\right]\right\} \mathrm{d} b_{2} .
\end{aligned}
$$

Note that $0 \leq \int_{0}^{v_{2} \varepsilon} \frac{b_{2} / \nu_{2}}{\varepsilon}\left(1-\delta / v_{1}\right) \mathrm{d} b_{2}<\frac{\varepsilon / \nu_{2}}{\varepsilon}\left(1-\delta / v_{1}\right) \cdot\left[v_{2} \varepsilon-0\right]=\left(1-\delta / v_{1}\right) \cdot \varepsilon$. Therefore $\int_{0}^{v_{2} \varepsilon} \frac{b_{2} / v_{2}}{\varepsilon}\left(1-\delta / v_{1}\right) \mathrm{d} b_{2}$ converges to zero when $\varepsilon \rightarrow 0^{+}$.

Note $\int_{v_{2} \varepsilon}^{v_{2}}\left\{\left(1-\delta / v_{1}\right)+\frac{\delta / v_{1}}{1-\varepsilon}\left[b_{2} / v_{2}-\varepsilon\right]\right\} \mathrm{d} b_{2}$ converges to $\int_{0}^{v_{2}} \mathrm{~d} b_{2}=v_{2}$ when $\varepsilon \rightarrow 0^{+}$and $\delta \rightarrow 0^{+}$ 
Therefore, $\operatorname{Pr}$ (bidder 2 wins) converges to 0 when $\varepsilon \rightarrow 0^{+}$and $\delta \rightarrow 0^{+}$.

In Figure 1, we illustrate the equilibrium bidding strategies of the players with small $\varepsilon$ and $\delta$. With small $\varepsilon$ and $\delta, B(\cdot)$ takes very small value whenever $b_{1} \leq v_{1}-\delta$, which means player 2 is extremely favored. We can see that player 1 places a bid higher than $v_{1}-\delta$ with probability $1-\varepsilon$, and that player 2 places a bid lower than $v_{2} \varepsilon$ with probability $1-\frac{\delta}{v_{1}}$. Therefore, in the limit we have the following results: player 1 always wins; player 1's expected effort must be $v_{1}$ and player 2's expected effort must be zero.

It is clear that the limit of bidding strategies in (4) when $\varepsilon$ and $\delta$ converge to zero do not form an equilibrium, so strictly speaking the optimal favoritism rule does not exist. However, an epsilon-optimality can be achieved by using a favoritism rule in (2) when $\varepsilon$ and $\delta$ are sufficiently small.

Such nonlinear favoritism rule in (2) favors bidder 2 extremely to incentivize bidder 1, i.e. the stronger bidder, to bid close to his/her value; at the same time, at the equilibrium, bidder 2 wins with nearly probability 0 even $s /$ he is extremely favored. Note that in a standard all pay auction, the weaker player wins with probability $\frac{v_{2}}{2 v_{1}}(>0)$ at equilibrium. Note that the bidding equilibrium in the standard all pay auction without favoritism can be identified by setting $B_{1}\left(b_{2}\right)$ and $B^{-1}\left(b_{1}\right)$ as identity functions in Proposition 1.

For the above identified optimal favoritism rule in (2), we have that at the limit, the stronger player wins with probability 1 , and both players have zero expected payoff. We claim these properties must be satisfied by any optimal favoritism rule,

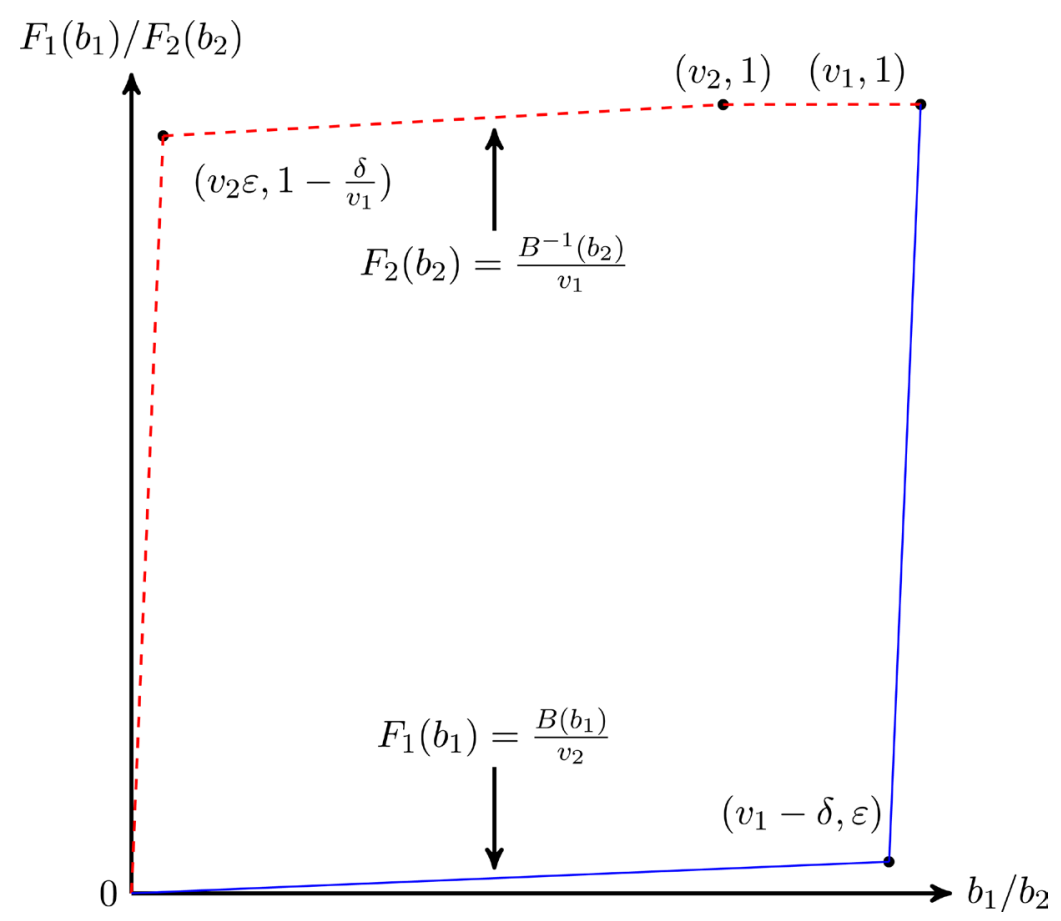

Figure 1. Illustration of Mixed Equilibrium Bidding Strategies. 
which induces the upper bound effort $v_{1}$, identified in Proposition 2.

Let $T E_{i}, i=1,2$ denote player is expected effort. Note player is expected payoff is

$$
\pi_{i}=v_{i} \operatorname{Pr}(\text { bidder } i \text { wins })-T E_{i} \text {. }
$$

Therefore, the total expected effort

$$
\begin{aligned}
& T E_{1}+T E_{2} \\
& =v_{1}[1-\operatorname{Pr}(\text { bidder } 2 \text { wins })]+v_{2} \operatorname{Pr}(\text { bidder } 2 \text { wins })-\left[\pi_{1}+\pi_{2}\right] \\
& =v_{1}+\left[v_{2}-v_{1}\right] \operatorname{Pr}(\text { bidder } 2 \text { wins })-\left[\pi_{1}+\pi_{2}\right] .
\end{aligned}
$$

Note that players' participation constraints mean that we must have $\pi_{i} \geq 0$. To have $T E_{1}+T E_{2}=v_{1}$, we must have $\operatorname{Pr}($ bidder 2 wins $)=0$ and $\pi_{i}=0$.

\section{A Geometric Interpretation}

Next, we present a geometrical interpretation of the logic behind the above identified optimal design. Consider any bidding equilibrium of Proposition 1. Player 1 's expected effort is

$$
\begin{aligned}
T E_{1} & =\int_{0}^{\bar{b}_{1}} b_{1} d F_{1}\left(b_{1}\right)=\int_{0}^{\bar{b}_{1}} b_{1} d \frac{B\left(b_{1}\right)}{v_{2}} \\
& =\left.\left[b_{1} \frac{B\left(b_{1}\right)}{v_{2}}\right]\right|_{0} ^{\bar{b}_{1}}-\frac{1}{v_{2}} \int_{0}^{\bar{b}_{1}} B\left(b_{1}\right) \mathrm{d} b_{1} \\
& =\bar{b}_{1}-\frac{1}{v_{2}} \int_{0}^{\bar{b}_{1}} B\left(b_{1}\right) \mathrm{d} b_{1},
\end{aligned}
$$

and player 2's expected effort is

$$
\begin{aligned}
T E_{1} & =\int_{0}^{v_{2}} b_{2} \mathrm{~d} F_{2}\left(b_{2}\right)=\int_{0}^{v_{2}} b_{2} \mathrm{~d}\left[\frac{v_{1}-\bar{b}_{1}}{v_{1}}+\frac{B^{-1}\left(b_{2}\right)}{v_{1}}\right] \\
& =b_{2}\left[\frac{v_{1}-\bar{b}_{1}}{v_{1}}+\frac{B^{-1}\left(b_{2}\right)}{v_{1}}\right]_{0}^{v_{2}}-\int_{0}^{v_{2}}\left[\frac{v_{1}-\bar{b}_{1}}{v_{1}}+\frac{B^{-1}\left(b_{2}\right)}{v_{1}}\right] \mathrm{d} b_{2} \\
& =v_{2}-\frac{v_{1}-\bar{b}_{1}}{v_{1}} v_{2}-\frac{1}{v_{1}} \int_{0}^{v_{2}} B^{-1}\left(b_{2}\right) \mathrm{d} b_{2} \\
& =\frac{v_{2}}{v_{1}} \bar{b}_{1}-\frac{1}{v_{1}} \int_{0}^{v_{2}} B^{-1}\left(b_{2}\right) \mathrm{d} b_{2} .
\end{aligned}
$$

Therefore, we have total expected effort

$$
T E_{1}+T E_{2}=\bar{b}_{1}+\frac{v_{2}}{v_{1}} \bar{b}_{1}-\left[\frac{1}{v_{2}} \int_{0}^{\bar{b}_{1}} B\left(b_{1}\right) \mathrm{d} b_{1}+\frac{1}{v_{1}} \int_{0}^{v_{2}} B^{-1}\left(b_{2}\right) \mathrm{d} b_{2}\right] .
$$

Note that $\int_{0}^{\bar{b}_{1}} B\left(b_{1}\right) \mathrm{d} b_{1}+\int_{0}^{v_{2}} B^{-1}\left(b_{2}\right) \mathrm{d} b_{2}$ is simply the area of $\left[0, \bar{b}_{1}\right] \times\left[0, v_{2}\right]$. Thus,

$$
\underbrace{\int_{0}^{\bar{b}_{1}} B\left(b_{1}\right) \mathrm{d} b_{1}}_{X}+\underbrace{\int_{0}^{v_{2}} B^{-1}\left(b_{2}\right) \mathrm{d} b_{2}}_{Y}=\bar{b}_{1} v_{2}, \forall B(\cdot), \text { s.t.: } B(0)=0, B\left(\bar{b}_{1}\right)=v_{2} .
$$


Since $\frac{1}{v_{2}}>\frac{1}{v_{1}}$, to maximize $T E_{1}+T E_{2}$, we want to minimize $\int_{0}^{\bar{b}_{1}} B\left(b_{1}\right) \mathrm{d} b_{1}$.

This means that we should set $X=\int_{0}^{\bar{b}_{1}} B\left(b_{1}\right) \mathrm{d} b_{1}=0$ and $Y=\int_{0}^{v_{2}} B^{-1}\left(b_{2}\right) \mathrm{d} b_{2}=\bar{b}_{1} v_{2}$ at the limit. This in turn requires that $B\left(b_{1}\right)$ takes very low value when $b_{1}$ is lower than $\bar{b}_{1}$.

As a result, in the limit, we have $\frac{1}{v_{2}} X+\frac{1}{v_{1}} Y=\frac{\bar{b}_{1} v_{2}}{v_{1}}$ at the optimum. Thus,

$$
T E_{1}+T E_{2}=\bar{b}_{1}+\frac{v_{2}}{v_{1}} \bar{b}_{1}-\frac{\overline{b_{1}} v_{2}}{v_{1}}=\overline{b_{1}},
$$

which means that to maximize $T E_{1}+T E_{2}$, we should further set $\bar{b}_{1}^{*}=v_{1}$ at optimum.

For this optimal $\bar{b}_{1}^{*}=v_{1}$, the identified favoritism rule $B\left(b_{1}\right)$ in (2) indeed makes $\int_{0}^{\bar{b}_{1}^{*}} B\left(b_{1}\right) \mathrm{d} b_{1}=\int_{0}^{v_{1}} B\left(b_{1}\right) \mathrm{d} b_{1}$ close to zero as $B\left(b_{1}\right)$ takes very low value when $b_{1}$ is lower than $v_{1}-\delta$

\section{Concluding Remarks}

Asymmetric competitions are everywhere. Introducing favoritism in these competitions is an effective way to mitigate the discouragement effect due to the heterogeneity of players. The literature on favoritism in asymmetric competitions has so far been focusing on linear instruments such as head-start and handicap. In this paper, we generalize the analysis to accommodate fully nonlinear favoritism rules, and explicitly characterize the effort-maximizing favoritism rule and the induced equilibrium in an analytical framework of all pay auctions with complete information. Our optimal design provides further guidance on how to better incentivize many asymmetric competitions in economic, political, social, and athletic contexts.

Besides effort elicitation, winner diversity is also one important consideration as evidenced by the rationale for the introduction of affirmative actions in school admissions, etc. Our findings reveal that favoritism focusing on pure effort-maximization can rather hurt the diversity compared to a standard all pay auction without favoritism. This result indicates that policy makers need to exercise caution when designing desirable favoritism rules. One possible extension of our study is to consider an objective of weighted average of effort supply and winner diversity. We leave this to future work.

In this paper, we adopt a technology of all pay auction in our analysis. Our main results crucially rely on this model structure, which entails mixed-strategy equilibria that is determined by the indifference conditions. Extending the analysis to a Tullock contest technology is an interesting but nontrivial exercise. We also leave this to future work.

\section{Acknowledgements}

I am very grateful to Professor Sérgio Parreiras for his advice on this research 
project. I thank an anonymous reviewer for his/her insightful comments and suggestions which significantly improved the quality of the paper.

\section{Conflicts of Interest}

The author declares no conflicts of interest regarding the publication of this paper.

\section{References}

Brown, J. (2011). Quitters Never Win: The (Adverse) Incentive Effects of Competing with Superstars. Journal of Political Economy, 119, 982-1013.

https://doi.org/10.1086/663306

Che, Y.-K., \& Gale, I. L. (1998). Caps on Political Lobbying. American Economic Review, $88,643-651$.

Chowdhury, S. M., Esteve-González, P., \& Mukherjee, A. (2019). Heterogeneity, Leveling the Playing Field, and Affirmative Action in Contests. Working Paper.

https://doi.org/10.2139/ssrn.3655727

Dragov, M., \& Ryvkin, D. (2017). Biased Contests for Symmetric Players. Games and Economic Behaviour, 103, 116-144. https://doi.org/10.1016/j.geb.2016.02.003

Dragov, M., \& Ryvkin, D. (2020). Hunting for the Discouragement Effect in Contests. Working Paper.

Epstein, G. S., Mealem, Y., \& Nitzan, S. (2011). Political Culture and Discrimination in Contests. Journal of Public Economics, 95, 88-93.

https://doi.org/10.1016/j.jpubeco.2010.09.012

Franke, J. (2012). Affirmative Action in Contest Games. European Journal of Political Econo$m y, 28,105-118$. https://doi.org/10.1016/j.ejpoleco.2011.07.002

Franke, J., Kanzow, C., Leininger, W., \& Schwartz, A. (2013). Effort Maximization in Asymmetric Contest Games with Heterogeneous Contestants. Economic Theory, 52, 589-630. https://doi.org/10.1007/s00199-011-0657-Z

Franke, J., Leininger, W., \& Wasser, C. (2018). Optimal Favoritism in All-Pay Auctions and Lottery Contests. European Economic Review, 104, 22-37.

https://doi.org/10.1016/j.euroecorev.2018.02.001

Fu, Q. (2006). A Theory of Affirmative Action in College Admissions. Economic Inquiry, 44, 420-428. https://doi.org/10.1093/ei/cbj020

Kirkegaard, R. (2012). Favoritism in Asymmetric Contests: Head Starts and Handicaps. Games and Economic Behavior, 76, 226-248. https://doi.org/10.1016/i.geb.2012.04.005

Lee, S. H. (2013). The Incentive Effect of a Handicap. Economics Letters, 118, 42-45. https://doi.org/10.1016/j.econlet.2012.09.024

Nti, K. O. (2004). Maximum Efforts in Contests with Asymmetric Valuations. European Journal of Political Economy, 20, 1059-1066. https://doi.org/10.1016/j.ejpoleco.2003.11.003

Pastine, I., \& Pastine, T. (2012). Student Incentives and Preferential Treatment in College Admissions. Economics of Education Review, 31, 123-130.

https://doi.org/10.1016/j.econedurev.2011.09.005

Sahuguet, N. (2006). Caps in Asymmetric All-Pay Auctions with Incomplete Information. Economics Bulletin, 3, 1-8.

Seel, C., \& Wasser, C. (2014). On Optimal Head Starts in All-Pay Auctions. Economics Letters, 124, 211-214. https://doi.org/10.1016/j.econlet.2014.05.018 
Tsoulouhas, T., Knoeber, C. R., \& Agrawal, A. (2007). Contests to Become CEO: Incentives, Selection and Handicaps. Economic Theory, 30, 195-221.

https://doi.org/10.1007/s00199-005-0060-8

Zhu, F. (2019). On Optimal Favoritism in All-Pay Contests. Working Paper. 